\title{
Comorbidité lèpre et tuberculose: à propos de six cas
}

\section{Comorbidity leprosy and tuberculosis: With reference to six cases} \author{
Diop S.A ${ }^{5}$, Touré-Badiane N. ${ }^{3}$, Dieng M.T ${ }^{4}$, Diop B.M ${ }^{5}$, Ka M.M ${ }^{1}$. \\ 1. Dermatologie, Université de Thiès, Sénégal. \\ 2. Centre Hospitalier de l'Ordre de Malte, Fann, Dakar, Sénégal. \\ 3. Pneumologie, Université Cheikh Anta Diop, Sénégal \\ 4. Dermatologie, Université Cheikh Anta Diop, Dakar, Sénégal. \\ 5. Maladies Infectieuses, Université de Thiès, Sénégal.
}

Dioussé $\mathbf{P}^{1}$, Fall L ${ }^{2}$, Lawson A.T.D ${ }^{5}$, Diop M.M ${ }^{1}$, Diop C.T ${ }^{3}$, Dione H. ${ }^{1}$, Bammo $\mathrm{M}^{1}$, Seck $\mathrm{F}^{1}$, Gueye A.B ${ }^{2}$,

Correspondance : Dr DIOUSSE Pauline Département de Maladies Infectieuses-Dermatologie-Vénérologie, UFR des sciences de la Santé, Université de Thiès, Email : paudiousse@yahoo.fr

\section{Résumé}

Objectif : Rapporter 6 observations de co-infection de la lèpre et de la tuberculose. Méthodes: Il s'agissait d'une série rétrospective transversale à visée descriptive réalisée au Centre Hospitalier de l'Ordre de Malte à Dakar (Sénégal) sur une période de 15 ans (2001-2016).Résultats: Six dossiers étaient étudiés. La moyenne d'âge des patients était de 29 ans $\pm 10,4$ [15 - 42 ans] avec un sex ratio $\mathrm{H} / \mathrm{F}$ de 2 ( 4 hommes pour 2 femmes). Quatre patients avaient une lèpre lépromateuse et deux une lèpre borderline (boderline lépromateuse). Le délai médian d'évolution entre la tuberculose et la lèpre était de 32 mois [5 mois- 48 mois]. Les formes de tuberculose retrouvées étaient des formes purement pulmonaires dans 4 cas et multifocale (pleural et ganglionnaire; pleural puis neurologique) dans 2 cas. Les facteurs de risque de survenue de la tuberculose étaient la corticothérapie générale au long cours, la malnutrition, l'anémie, le tabagisme et la grossesse. La sérologie antirétrovirale à VIH était négative chez tous les patients. Les traitements de la lèpre et de la tuberculose étaient prescrits selon les protocoles de l'OMS. Tous les patients étaient déclarés guéris de la lèpre et de la tuberculose.Conclusion : la survenue de la lèpre dans sa forme multibacillaire sur un terrain immunitaire déficitaire pourrait favoriser soit la réactivation d'une tuberculose latente sous-jacente ou une surinfection de la lèpre par Mycobacterium tuberculosis. Il paraît donc important de rechercher activement la tuberculose durant le screening des patients atteints de lèpre surtout lépromateuse.

Mots clés : Lèpre, Tuberculose, Co-infection, Dakar, Sénégal.

\footnotetext{
Abstract

Purpose: Report six cases of Leprosy and Tuberculosis Co-infection.Procedure: This was a series of retrospective cross-sectional descriptive carried out at the Hospital Center of the Order of
}

Malta in Dakar (Senegal) over a period of 15 years (2001-2016).Results: Six cases were studied. The mean age of the patients was 29 years \pm 10.4 [15-42 years] with a sex ratio $\mathrm{H} / \mathrm{F}$ of 2 (4 men for 2 women). Four patients had lepromatous leprosy and two had borderline leprosy (lepromatous boderline). The median time to progression between tuberculosis and leprosy was 32 months $[5$ months48 months]. The forms of tuberculosis found were purely pulmonary forms in 4 cases and multifocal (pleural and ganglionic, pleural then neurological) in 2 cases. The risk factors for the occurrence of tuberculosis were long-term systemic steroids, malnutrition, anemia, smoking and pregnancy. HIV antiretroviral serology was negative in all patients. The treatment of leprosy and tuberculosis was prescribed according to WHO protocols. All patients were declared cured of leprosy and tuberculosis. Conclusion: the occurrence of leprosy in its multibacillary form on a deficit immune field could favor either the reactivation of an underlying latent tuberculosis or a superinfection of leprosy by Mycobacterium tuberculosis. It therefore seems important to actively search for tuberculosis during the screening of leprosy patients, especially lepromatous patients.

Key words: Leprosy, Tuberculosis, Co-infection, Dakar, Senegal.

\section{Introduction}

La lèpre et la tuberculose sont deux maladies infectieuses dues respectivement à des bacilles intracellulaires acido-alcoolo-résistants (BAAR): Mycobacterium leprae et $M$. tuberculosis [1, 2]. Selon l'Organisation Mondiale de la Santé (OMS), en 2015, en Afrique, le nombre de nouveaux cas de lèpre et de tuberculose était respectivement de 210000 et 8,6 millions $[3,4]$. La lèpre et la tuberculose demeurent endémiques au Sénégal avec des taux d'incidence respectivement de moins de 1 cas pour 10000 habitants et 139 cas pour 100000 habitants [5]. Ces deux infections chroniques 
granulomateuses sont contrôlées par une réponse immunitaire à médiation cellulaire [1-6]. Le risque de développer une tuberculose active est beaucoup plus élevé en cas de déficience du système immunitaire comme lors de traitements immunosuppresseurs comme la corticothérapie au long cours. La co-infection lèpre et tuberculose est rarement décrite dans la littérature. Une étude portant sur les similitudes entre l'immunité des deux mycobactéries a été menée au Sénégal [712].Nous rapportons 6 observations de co-infection pour les 2 mycobactéries.

\section{Méthodes}

Il s'agit d'une série rétrospective transversale à visée descriptive réalisée au Centre Hospitalier de l'Ordre de Malte à Dakar sur une période de 15 ans, soit du 1 janvier 2001 au 31 décembre 2016. Etaient inclus tous les dossiers de patients reçus en consultation externe pour une lèpre clinique, bactériologique et histologique ayant développé une tuberculose clinique, bactériologique et radiologique. Les dossiers non exploitables n'étaient pas retenus. Les données étaient recueillies et analysées par Microsoft Excel version 2010. Nous avons reçu un avis favorable du comité d'éthique de l'université, nous avons obtenu au préalable l'accord du chef de service et nous avons garanti l'anonymat des patients. Nous ne déclarons aucun conflit d'intérêt.

\section{Résultats}

Nous avons retenus 6 cas sur 480 dossiers colligés soit une fréquence de $1,25 \%$. L'âge moyen des patients était de 29 ans 29 ans $\pm 10,4$ [15 - 42 ans] avec un sex ratio $\mathrm{H} / \mathrm{F}$ de 2 (4 hommes pour 2 femmes). L'antécédent de contage lépreux existait dans un cas et celle de contage tuberculeux aussi dans un cas. Le délai Le délai médian d'évolution pour les deux infections était de 32 mois [5 mois48 mois]. La première infection était la lèpre dans 5 cas et la tuberculose dans 1 cas. Les formes cliniques de lèpre étaient lépromateuselépromateuse dans 4 cas et borderline-lépromateuse dans 2 cas. Tous les patients avaient une hypertrophie de plusieurs troncs nerveux avec un déficit sensitif et moteur. Cinq patients avaient des réactions lépreuses de type 2 . Les formes de tuberculose retrouvées étaient des formes purement pulmonaires dans 4 cas et multifocale (pleurale et ganglionnaire; pleurale puis neurologique) dans 2 cas (Figures 1 - 6). Les facteurs favorisants pour la survenue de la tuberculose étaient la corticothérapie générale au long cours, la malnutrition, l'anémie, le tabagisme et la grossesse. La sérologie antirétrovirale à VIH était négative chez tous les patients. Le résumé clinique et para-clinique est décrit au tableau (tableau 1). Les traitements de la lèpre et de la tuberculose étaient prescrits selon les protocoles de l'Organisation Mondiale de la Santé simultanément dans 2 cas et après le traitement anti lépreux dans 4 cas. Tous les patients étaient déclarés guéris de la lèpre et de la tuberculose après les traitements spécifiques et plusieurs contrôles cliniques, bactériologiques et radiologiques.

\section{Discussion}

Le présent travail rapporte 6 cas de co-infection lèpre et tuberculose. Ces deux maladies sévissent à l'état endémique au Sénégal. La première n'est plus un problème de santé publique mais pose un problème actuel de réémergence la seconde reste toujours une préoccupation sur le plan sanitaire [13]. Dans notre série comme dans certaines observations, la lèpre avait vraisemblablement précédé la tuberculose. Elle avait suivi la tuberculose dans la série de Sendrasoa et al. [8]. L'écart entre le développement de la lèpre et la tuberculose variait de 5 mois et 04 ans dans notre série, elle variait entre deux mois à 10-15 ans dans d'autres et les deux maladies sont concomitantes dans certains travaux [7, 9-11, 14]. Dans tous les cas, le principal mode de transmission à la fois de la lèpre et de la tuberculose est la voie aérienne. La période d'incubation de la lèpre varie de neuf mois à 20 ans ou plus. L'évolution de la tuberculose comprend deux temps d'incubation distincts. Le premier concerne la primo-infection (4 à 12 semaines): c'est le temps séparant le contact avec le bacille de Koch, aussi appelé Mycobacterium tuberculosis et le virage positif des tests tuberculiniques, ce temps est asymptomatique. Une fois cette primo-infection faite, le deuxième temps d'incubation peut être bref (immédiat) ou long (jusqu'à plusieurs années), avant l'apparition de la tuberculose et des premiers symptômes [1, 3]. Toutefois, un organisme affaibli réduit la capacité du système immunitaire à réagir suffisamment ou assez rapidement, ainsi un micro-organisme plus virulent peut infecter le patient. Tous les patients présentaient une lèpre lépromateuse (Boderline Lépromateuse ou Lépromateuse Lépromateuse). Dans la lèpre multibacillaire (lépromateuse), les patients présentent une altération de la réponse à médiation cellulaire à Mycobacterium leprae, qui est supposée être associée au type HLA selon Abel et al. [15]. Il peut également être lié à un défaut dans le récepteur de type toll 2 (TLR2), qui est une protéine qui jouerait un rôle vital dans le déclenchement des mécanismes de défense de l'hôte contre l'invasion microbienne. Cette altération de l'immunité associée à la présence de certains facteurs favorisants chez les patients (la malnutrition, le tabagisme, la grossesse et l'anémie) pourrait expliquer, en partie, la baisse de la réponse immunitaire [16]. Il est bien connu que la tuberculose peut se développer du fait de l'infection par le VIH, du faible statut socio-économique, du diabète, de l'insuffisance rénale, de la corticothérapie au long cours et des traitements immunosuppresseurs. Dans la lèpre, les 
corticostéroïdes sont utilisés principalement dans le traitement des réactions sévères de types I et II et des neuropathies silencieuses. Tous les patients sauf dans un cas, étaient sous corticothérapie au long cours. Chandrashekhar et al a rapporté le développement de la tuberculose pulmonaire après la prise de corticoïdes dans deux cas de lèpre et Agarwal et al. [17] a rapporté un cas de coinfection lèpre et tuberculose chez un patient de receveur de greffe rénale et qui était sous prednisolone, azathiopirine et cyclosporine pendant plus de neuf ans $[1,18]$. Du fait de l'immunité croisée, certains auteurs ont affirmé que les patients ayant une tuberculose guérie étaient protégés contre le bacille de Hansen ; le vaccin BCG conférerait une certaine protection contre la lèpre. L'effet protecteur du BCG contre la lèpre est transitoire mais prouvé, ce qui a même amené les brésiliens à maintenir cette vaccination obligatoire dans leur programme de lutte contre la lèpre. Launois et al a montré que malgré la similitude de $90 \%$ entre les protéines $85 \mathrm{~A}$ de Mycobacterium leprae et Mycobacterium tuberculosis, certaines peptides étaient différents. Il a aussi démontré que la stimulation des cellules $\mathrm{T}$ (les régions 41 à 80 et 241 à 295), donnaient lieu à des réponses prolifératives puissantes et à une sécrétion d'IFNgamma. Parmi les cas rapportés, quatre présentaient une tuberculose pulmonaire comme dans la littérature et deux cas de tuberculose multifocale. Certains auteurs ont décrit des cas d'association lèpre et tuberculose cutanée [8-10, 12, 17, 19]. Sur le plan thérapeutique, les protocoles antituberculeux et anti- lépreux de l'Organisation Mondiale de la Santé étaient prescrits chez tous les patients. En plus, il avait été signalé une mauvaise observance à la polychimiothérapie anti-lépreuse chez 3 patients. Et cela était dû à une utilisation irrégulière de la rifampicine. Cette molécule est aussi un antituberculeux bactéricide de première intention qui, mal utilisé, peut contribuer au développement de la pharmaco-résistance acquise et réduire l'efficacité du traitement antituberculeux et, la prévalence de la tuberculose multi-résistante est déjà très élevée (11\%) au Sénégal [20].

\section{Conclusion}

La survenue de la lèpre dans sa forme multibacillaire sur un terrain immunitaire déficitaire pourrait favoriser soit la réactivation d'une tuberculose latente sous-jacente ou une surinfection de la lèpre par Mycobacterium tuberculosis. Il paraît donc important de rechercher activement la tuberculose durant le screening des patients atteints de lèpre surtout lépromateuse. Les programmes nationaux de lutte contre ces deux maladies doivent travailler en synergie d'autant plus que certains médicaments spécifiques sont identiques et les moyens de prévention étant presque les mêmes.

\section{Références}

1. Aubry $P$, Gaüzère BA. Lèpre ou Maladie de Hansen. Médecine tropicale, Actualités 2016. Mise à jour le 09/09/2017. p1-12. www.medecinetropicale.com

2. Yombi JC, Olinga UN. La tuberculose : épidémiologie, aspect clinique et traitement. Louvain Med 2015; 134 (10): 549-59.

3. Organisation Mondiale de la Santé. Situation de la lèpre dans le monde, 2015: l'heure est à l'action, à la responsabilisation et à l'inclusion. Relevé épidémiologique hebdomadaire /WER 2016; 35(91) : 405-20. http://www.who.int/wer. Consulté le $25 / 06 / 2017$

4. Organisation Mondiale de la Santé. Rapport sur la lutte contre la tuberculose dans le monde. 2016 : $1-6$.

http://www.who.int/entity/tb/publications/global re port/gtbr2016 executive summary fr.pdf. Consulté le $25 / 06 / 2017$

5. Ministère de la Santé et de l'Action Sociale du Sénégal. Programme National d'Elimination de la Lèpre. $\quad$ http://www.sante.gouv.sn/page-readerprojets-programmes-details. Consulté le 30/06/2017 6. OMS Tuberculose: profils de pays (Sénégal). Mise à jour le 28/12/2016. http://www.who.int/tb/country/data/profiles/fr/

Consulté le 30/06/2017

7. Trindade MAB, Miyamoto D, Benard G, SakaiValente NY, Vasconcelos DDM, Naafs B. Leprosy and tuberculosis co-infection: clinical and immunological report of two cases and review of the literature. American Journal of Tropical Medicine and Hygiene 2013; 88 (2): 236-40.

8. Sendrasoa FA, Ranaivo IM, Raharolahy O, Andrianarison M, Ramarozatovo LS, Rapelanoro Rabenja F. Pulmonary Tuberculosis and Lepromatous Leprosy Coinfection. Case Reports. Dermatological Medicine 2015; volume: 1-4.

9. Andonaba JB, Barro-Traoré F, Kyélem CG, Kafando C, Diallo B, Niamba $\mathrm{P}$ et al. Comorbididité tuberculose pulmonaire et lèpre multibacillaire chez un adulte à Bobo-Dioulasso (Burkina Faso). Bull de l'ALLF 2012; 27: 15.

10. Prasad R, Verma SK, Singh R, Hosmane G. Concomitant pulmonary tuberculosis and borderline leprosy with type- II lepra reaction in single patient. Lung India 2010; 27 (1): 19-23.

11. Rawson TM, Anjum V, Hodgson J, Rao AK, Murthy K, Rao PS et al. "Leprosy and tuberculosis concomitant infection: a poorly understood, age-old relationship," Leprosy Review 2014; 85 (4): 288-95.

12. Launois P, DeLeys R, Niang MN, Drowart A, Andrien M, Dierckx P et al. T-cell-epitope mapping of the major secreted mycobacterial antigen Ag85A in tuberculosis and leprosy. Infect Immun 1994; 62 (9): 3679-87. 
13. Niang SO, Diallo M, Ndiaye M, Ly F, Diallo $\mathrm{M}$, Diop A et al. Epidemiologic and clinicopathologic aspects of leprosy in Dakar; evaluation of 73 new cases. Dermatology Reports 2011; 3 (18):40-2.

14. Sreeramareddy CT, Menezes RG, Kishore PV. Concomitant age old infections of mankindtuberculosis and leprosy: a case report. Journal of Medical Case Reports 2007; 5 (1): 43.

15. Abel L, Sanchez FO, Oberti J, Nguyen V, Le Van H, Lap V.D et al. Susceptibility to leprosy is linked to the human NRAMP1 gene. J. Infect Dis 1998; 177: 133-45.

16. Krutzik SR, Modlin RL. The role of toll-like receptors in combating mycobacteria. Sem Immunol 2004; 16: 35-41.

17. Chandrashekhar TS, Ritesh GM, Kishore PV. Concomitant age- old infections of mankind Figures et tableaux

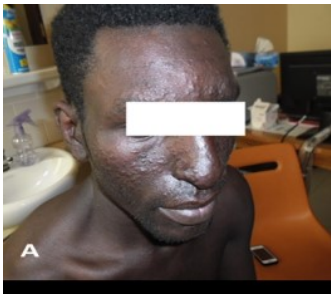

Fig 1: (A) Lésions lépromateuses du visage

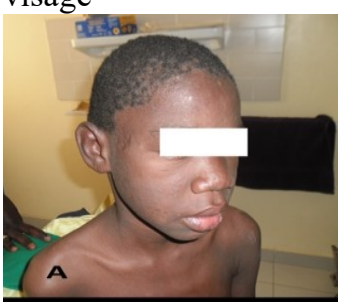

Fig 2: (A) Nodules infiltrés du visage

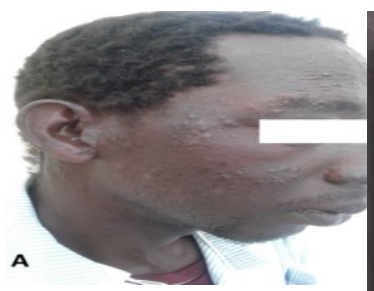

Fig 3: (A) Lésions nodulaires du visage

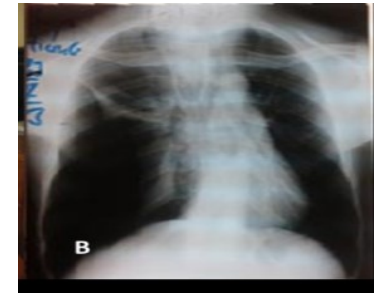

Fig 1: (B) Scissurite et pneumothorax à droite

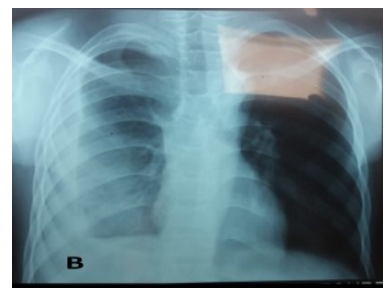

Fig 2 : (B) opacité basale droite

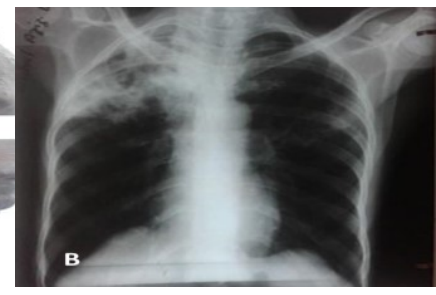

Fig 3: (B) foyer de condensation apicale droite

tuberculosis and leprosy: a case report. Lung India 2010; 27 (1): 19-23.

18. Agarwal DK, Mehta AR, Sharma AP, Sural S, Kumar A, Mehta B et al. Coinfection with leprosy and tuberculosis in a renal transplant recipient. Nephrol Dial Transplant 2000; 15: 1720-1.

19. Parise-Fortes MR, Lastória JC, Marques SA. Lepromatous leprosy and perianal tuberculosis: a case report and literature review. Journal of Venomous Animals and Toxins including Tropical Diseases 2014; 20 (38): 2-6.

20. Gehre F, Otu J, Kendall L, Forson A, Kwara A, Kudzawu $\mathrm{S}$ et al. The emerging threat of preextensively drug-resistant tuberculosis in West Africa: preparing for large-scale tuberculosis research and drug resistance surveillance. BMC Medicine 2016; 14 (1):160.
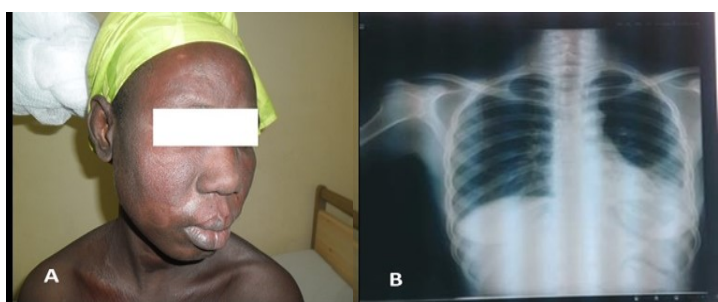

Fig 4: (A) Macules Fig 4: (B) foyer de infiltées du visage condensation basale

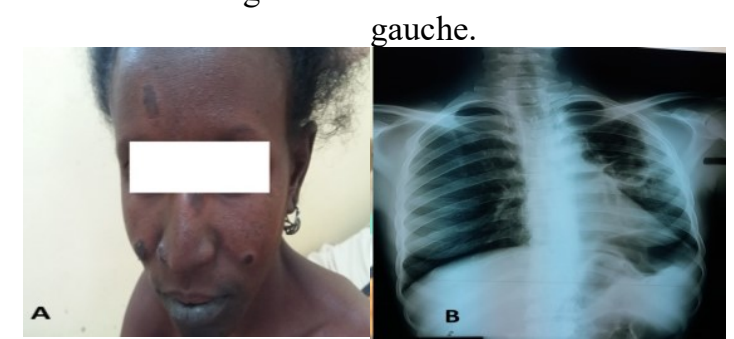

Fig 5: (A) Nodules Fig 5: (B) dilatation des infiltrés du visage bronches et atélectasie

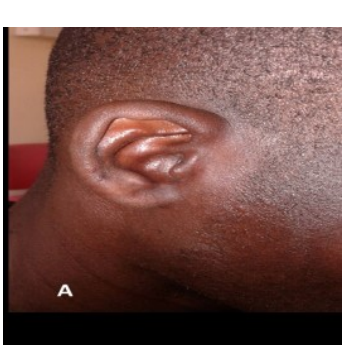

Fig 6: (A) Nodules des oreilles gauche.

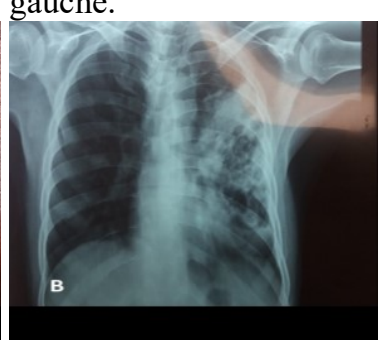

Fig 6: (B) foyer de condensation du champ pulmonaire gauche. 


\begin{tabular}{|c|c|c|c|c|c|c|}
\hline Observation & 1 & 2 & 3 & 4 & 5 & 6 \\
\hline Type de lèpre & LL & $\mathrm{LL}$ & $\mathrm{LL}$ & $\mathrm{BL}$ & $\mathrm{BL}$ & $\mathrm{LL}$ \\
\hline $\begin{array}{l}\text { Type de réaction } \\
\text { lépreuse }\end{array}$ & 2 & 2 & 2 & 2 & 1 & 2 \\
\hline Facteurs de risque & $\begin{array}{l}\text { Corticostéroïdes } \\
\text { Anémie, Malnutrition } \\
\text { Tabagisme }\end{array}$ & $\begin{array}{l}\text { Corticostéroïdes } \\
\text { Malnutrition }\end{array}$ & Corticostéroïdes & $\begin{array}{l}\text { Corticostéroïdes } \\
\text { Grossesse }\end{array}$ & Anémie Malnutrition & Corticoïdes \\
\hline Type de tuberculose & Pulmonaire & $\begin{array}{l}\text { Pleurale } \\
\text { Ganglionnaire }\end{array}$ & Pulmonaire & Pulmonaire & $\begin{array}{l}\text { Pulmonaire } \\
\text { Neurologique }\end{array}$ & Pulmonaire \\
\hline $\begin{array}{l}\text { Radiographie } \\
\text { thoracique }\end{array}$ & $\begin{array}{l}\text { Scissurite et pneumothorax } \\
\text { à droite }\end{array}$ & Opacité basale droite & $\begin{array}{l}\text { foyer de condensation } \\
\text { apicale droite }\end{array}$ & $\begin{array}{l}\text { foyer de condensation } \\
\text { basale gauche }\end{array}$ & $\begin{array}{l}\text { Dilatation des } \\
\text { bronches et } \\
\text { atélectasie gauche }\end{array}$ & $\begin{array}{l}\text { Foyer de } \\
\text { condensation } \\
\text { pulmonaire gauche }\end{array}$ \\
\hline $\begin{array}{l}\text { Confirmation de la } \\
\text { tuberculose }\end{array}$ & Crachats BAAR Positif & $\begin{array}{l}\text { Crachats BAAR } \\
\text { Négatif } \\
\text { Gene-Xpert positif }\end{array}$ & $\begin{array}{l}\text { Crachats BAAR Positif à } \\
2 \text { croix }\end{array}$ & $\begin{array}{l}\text { BAAR non fait } \\
\text { Gene-Xpert crachats } \\
\text { positif }\end{array}$ & $\begin{array}{l}\text { Crachats BAAR } \\
\text { Positif à } 2 \text { croix }\end{array}$ & $\begin{array}{l}\text { Crachats BAAR } \\
\text { Positif à } 1 \text { croix }\end{array}$ \\
\hline $\begin{array}{l}\text { Confirmation de la } \\
\text { lèpre }\end{array}$ & $\begin{array}{l}\text { Frottis cutané }+++ \\
\text { histopathologie } \\
\text { contributive }\end{array}$ & $\begin{array}{l}\text { Frottis cutané }+ \\
\text { histopathologie } \\
\text { contributive }\end{array}$ & $\begin{array}{l}\text { Frottis cutané }+++++ \\
\text { histopathologie } \\
\text { contributive }\end{array}$ & $\begin{array}{l}\text { Frottis cutané }+++ \\
\text { histopathologie } \\
\text { contributive }\end{array}$ & $\begin{array}{l}\text { Frottis cutané - } \\
\text { histopathologie } \\
\text { contributive }\end{array}$ & $\begin{array}{l}\text { Frottis cutané - } \\
\text { histopathologie } \\
\text { contributive }\end{array}$ \\
\hline
\end{tabular}

